\section{Strategies for Preventing Detachment of Sections from Glass Slides}

J. A. Kiernan, The University of Western Ontario

A thin, flat section of animal or plant tissue will usually adhere to a clean glass surface and stay in place through the common preparative procedures of staining, washing, dehydration and clearing. The adhesion may be due largely to the close contact between two flat surfaces. This allows very weak non-ionic forces (such as van der Waals forces and hydrogen bonds) between individual atoms, to exist in numbers great enough to add up to a strong attraction between slide and section. lonic attractions, which operate over longer distances, may also exist between the silicate of the glass (negatively charged ions, especially in an alkaline medium) and basic groups of proteins in the tissue (positive ions, especially in an acidic medium). Unfortunately, not all sections are perfectly flat and not all slides are perfectly clean. Even when these conditions are met, there are some staining techniques that are harsh enough to remove the flattest section from the cleanest slide.

There are two ways to prevent or reduce the loss of sections subjected to harsh treatments: (a) by using an adhesive, and (b) by inclusion in a permeable protective film.

\section{Adhesives}

The most popular way to try to keep sections on slides is to coat the glass with an adhesive. Many kinds are available and some are briefly reviewed as follows. The efficiency of an adhesive depends on the nature of the treatment that might cause detachment of the sections.

\section{Adhesion of oppositely charged surfaces.}

When immersed in an aqueous medium in the $\mathrm{pH}$ range 5 to 7 , most animal tissues carry a net negative charge, owing to a small excess of acidic over basic amino acids in the structural proteins. Plant tissues are composed largely of cellulose, which does not itself form ions but is impregnated with a variety of weak acids, and these too confer an overall negative charge. Sections of almost any tissue can therefore be expected to adhere well to a glass surface that has been treated in such a way as to make it positively charged. The modification is usually accomplished either by coating the slide with a basic polymer or by a chemical reaction that leaves amino groups linked by covalent bonds to the silicon atoms of the glass.

A frequently used basic polymer is polylysine, in which every amino acid unit has an amino side chain that is quite strongly basic. Its $\mathrm{pK}_{\mathrm{a}}$ is 10.53 . This is the $\mathrm{pH}$ at which half the amino groups are protonated. The side-chain of lysine has an unusually high $\mathrm{pK}_{\mathrm{a}}$ for an amino group and consequently polylysine is positively charged even in moderately alkaline media. Polylysine is applied to slides as an aqueous solution, which is then allowed to dry by evaporation. Either D- or Lpolylysine or the somewhat less expensive racemic DL- mixture may be used because the stereochemistry does not affect the ionization of the side chain. The guanidine side-chain of arginine is a strong base $\left(\mathrm{pK}_{\mathrm{a}}=12.5\right)$. Polyarginine should therefore retain its positive charge even in the most strongly alkaline conditions, but it is even more expensive than polylysine and does not seem to have been used as a section adhesive.

The other way to make a positively charged glass surface is to treat it with a solution of 3-aminopropyltriethoxysilane (APES) in the presence of catalytic traces of water. The $0.1 \%$ or so of water ordinarily present in acetone is enough.

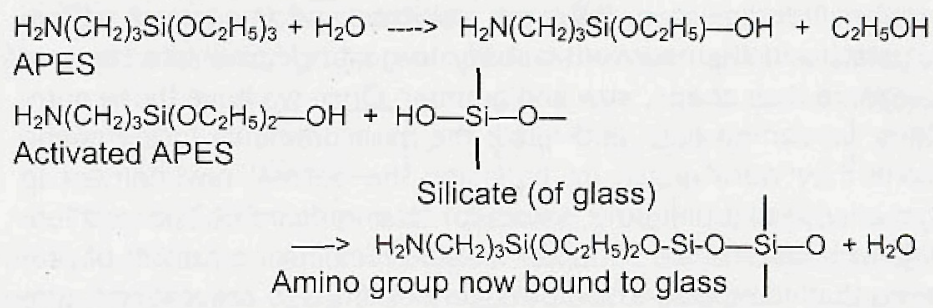

The positive charge of APES-treated (silanized) slides is like that imparted by polylysine, but covalent bonding to the glass ensures that it cannot be washed away.

Adhesion to a polylysine-coated or APES-treated slide depends on the protonation of the -- $\mathrm{NH}_{2}$ groups on the surface of the glass. An alkaline solution will suppress the formation of positive ions:

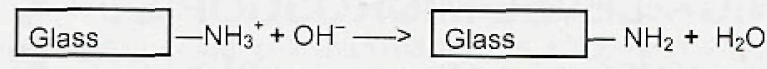

The reduced number of positively charged sites will diminish the efficacy of the sticking. On the other hand, an alkaline medium will enhance the ionization of carboxyl groups of protein sidechains:

Protein $-\mathrm{COOH}+\mathrm{OH}^{-} \rightarrow$ Protein $-\mathrm{COO}^{-}+\mathrm{H}_{2} \mathrm{O}$

This might or might not overcome the adverse effect of high $\mathrm{pH}$ on the amino groups attached to the slide. The only way to find out is by trial and error.

The carboxyl groups of proteins (and of some macromolecular carbohydrates) vary in their responses to $\mathrm{pH}$. All are weak acids: many remain ionized at $\mathrm{pH} 2.5$ but all are uncharged at $\mathrm{pH} 1$.

Some tissues (cartilage matrix is a notable example) contain an abundance of sulphate-ester groups. These are strong acids, ionized at any $\mathrm{pH}$. Tissues of this kind are therefore attracted to protonated amino groups however low the $\mathrm{pH}$, but will not be attracted by a negatively charged surface such as glass with its silicate ions or an adhesive coating that contains an excess of carboxyl groups.

Table 1 contains instructions for a simple procedure that attaches amino groups to the surfaces of glass slides.

\section{Gummy Adhesives}

Starch paste, poly(vinyl acetate) (white glue), egg albumen and gelatin are sticky substances composed of large molecules that can intervene between the opposed surfaces of the section and the slide, filling in any irregularities. They either dissolve or disperse in water, and if applied too thickly can increase rather than reduce the chance of losing sections. When egg albumen is used, it is usual to heat the slides to $55-60^{\circ} \mathrm{C}$, after mounting the sections. This coagulates the layer of albumen, which is then no longer soluble in water.

Adhesion to a layer of gelatin can be enhanced by exposing the slides to formaldehyde vapor (overnight at room temperature in a closed container). This cross-links the gelatin, making it insoluble. Formaldehyde may also form some covalent linkages between suitably placed nitrogen atoms in gelatin molecules and tissue proteins. Chrome gelatin provides another way to make use of strong chemical bonds.

\section{Continued on page 22}


$\gamma \mathbf{P} \mathbf{G} \mathbf{T}$

zancerono vaman atect
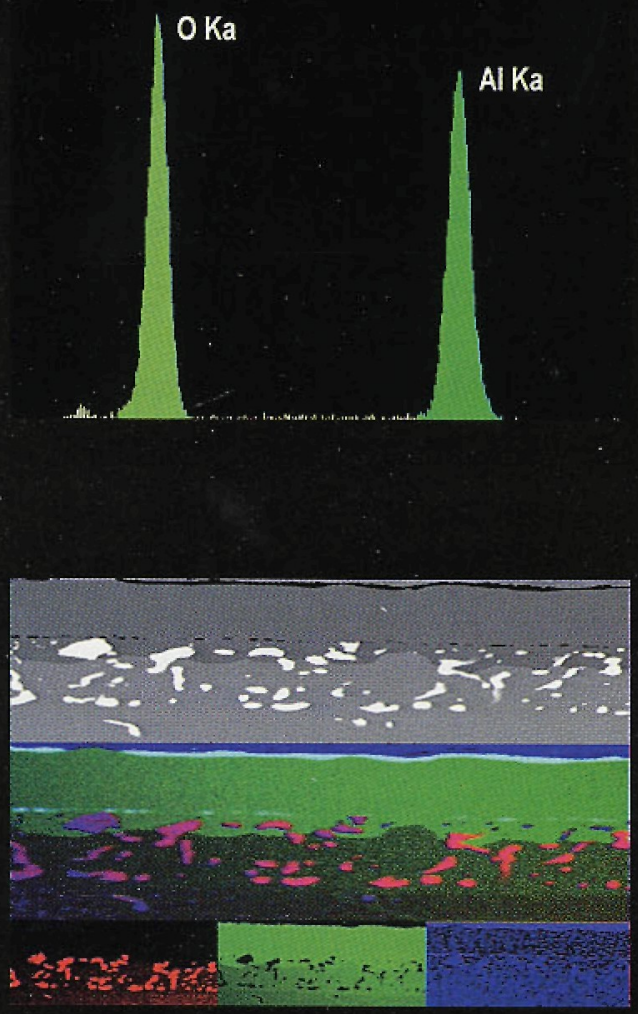

\section{Avalon 8000:}

\section{Workhorse for}

\section{everyday solutions!}

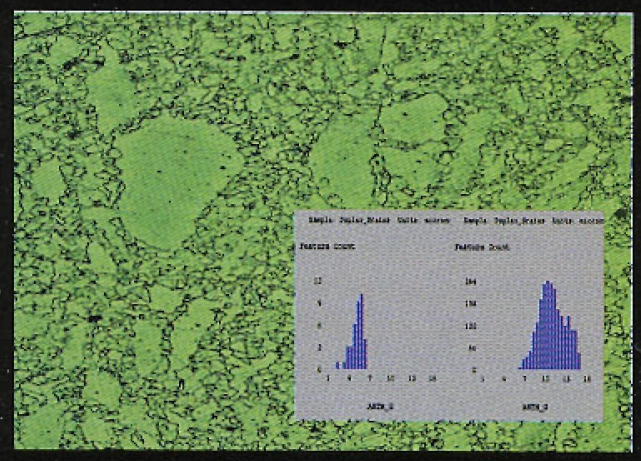

\section{IMIX:}

\section{Simply the best!}

For over 30 years you have come to PGT for the latest innovations in X-ray microanalysis and EDS detector technology.

Princeton Gamma-Tech, Inc.

C/N 863 Princeton, NJ 08542-0863

Telephone: (609) 924-7310

Facsimile: (609) 924-1729

e-mail: sales@pgt.com

website: www.pgt.com
Now you can come to us for inexpensive upgrades, fundamental workhorse systems, as well as solutions for advanced applications. 


\section{Strategies for Preventing Detachment of Sections from Glass Slides \\ Oontinued from page 20}

\section{Chrome Gelatin}

A coordinate bond is a special type of covalent bond formed between a metal ion and another atom, commonly oxygen or nitrogen. A single chromium (III) ion can form as many a six coordinate bonds. In an aqueous solution of a simple chromic salt the chromium (III) ions are joined by coordinate bonds to the oxygen atoms of water molecules. Strong coordinate bonds can form between chromium and the oxygen atoms of carboxyl groups of proteins. Advantage is taken of this in the process of chrome tanning. Chromium ions cross-link the carboxyl groups of collagen, converting them into leather. Tanning has much in common with histological fixation.

An adhesive for sections is prepared by mixing dilute aqueous solutions of gelatin and chromium potassium sulphate (chrome alum), which is an easily available chromium (III) salt. The resulting liquid has a consistency similar to that of nondrip paint, because the large molecules of gelatin are crosslinked by coordinate bonds to form, perhaps, a single huge molecule. The chrome gelatin cannot be further diluted with water once cross-linking has occurred, which seems to be within a few minutes of mixing the components. Chrome gelatin may be smeared onto glass slides with the end of a finger, or trays of slides may be dipped in the mixture for a few seconds. The treated slides are then allowed to air-dry in a dustfree place. Slides coated with chrome-gelatin are often called "subbed slides". They can be kept for at least three years before using. Sections are floated on water and collected onto subbed slides, which are then allowed to dry in the usual way. Cryostat sections can be collected onto the slides and allowed to thaw and dry. Thicker frozen sections can be mounted from water and adjusted with a paintbrush, but the stickiness of a subbed slide can make it difficult to straighten folds and wrinkles.

The mechanism of adhesion to a layer of chrome-gelatin is presumed to involve coordinate bonds between chromium ions in the adhesive and oxygen atoms in both the glass and the tissue:

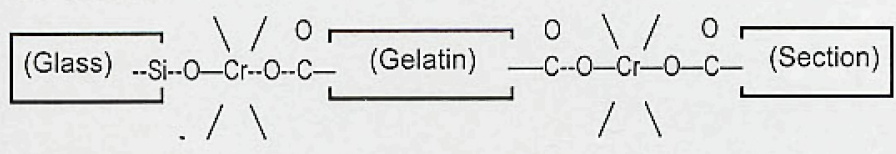

Table 1: Making Silanized Slides

\section{What you need:}

1. Slides - must be very clean and dry.

2. Staining racks to hold the slides

(glass or metal).

3. Staining tanks - one just large

enough to hold the staining rack, and one of larger size.

4. Water (distilled or deionized) - fill the larger staining tank.

5. Silanizing reagent. Add $4 \mathrm{~mL}$ of 3 aminopropyltriethoxysilane to $200 \mathrm{~mL}$ of acetone in the small staining tank. This solution must be used the day it is made.

The tank of silanizing solution can be used repeatedly and $200 \mathrm{~mL}$ will process hundreds of slides. boxes until they are needed. It is conventhey came in.

What to do:
1. Put the clean, dry slides in the stain-
ing rack.
2. Immerse in the silanizing solution for
30 to 60 seconds.
3. Shake off excess liquid and transfer
the rack of slides to a large tank of water.
Agitate, and leave for about one minute.
4. Remove the washed slides and put
them in a dust-free place until they are
dry.
The dry silanized slides are stored in
boxes until they are needed. It is conven-
ient to pack them in the cardboard boxes
they came in.

The short, oblique lines shown radiating from the chromium atoms represent coordinate bonds to other carboxyl groups of gelatin and to water molecules.

The formation of a coordinate bond between a chromium (III) atom and a carboxyl group requires that the latter be ionized:

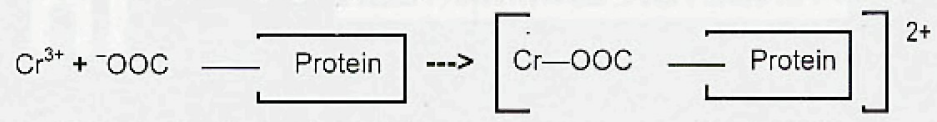

The ionization of protein carboxyl groups is largely suppressed if the ambient $\mathrm{pH}$ is below about 3 . Alkaline conditions, on the other hand, favor the formation of strong coordinate bonds to chromium. Consequently, chrome gelatin is particularly effective in preventing detachment of sections from slides during treatment with alkaline reagents.

The chrome-gelatin solution used for subbing (see Table 2) quickly becomes infected with micro-organisms. It is therefore made in the laboratory and used the same day.

\section{Protection with a Nitrocellulose Film}

Nitrocellulose (also called celloidin and collodion, and more correctly named cellulose nitrate) is soluble in ether-alcohol, a mixture of equal volumes of ethanol and diethyl ether. It swells but does not dissolve in ethanol, and is insoluble in $70 \%$ alcohol. Undissolved nitrocellulose readily forms a thin membrane, which is permeable to water, inorganic ions and smaller organic ions and molecules such as dyes, but not to macromolecules such as antibodies and other proteins. A properly applied layer of celloidin on top of the mounted sections on a slide can protect against losses even if there is failure of adhesion between the sections and the glass.

The slide bearing the sections must be completely enclosed by the nitrocellulose membrane, with no free edges that might come adrift during processing and staining. In this technique, described in Table 3 , the sections are passed through dehydrating solvents, so the method cannot be used in conjunction with some staining methods, such as those for lipids or most enzymatic activities.

\section{Continued on page 24}

Table 2: Making Subbed Slides

What you need:

1. Slides - must be clean and dry.

2. Staining racks to hold the slides (glass or metal).

3. Staining tank - just big enough to hold the staining rack.

4. Subbing solution:

Dissolve $0.3 \mathrm{~g}$ gelatin in $290 \mathrm{~mL}$ distilled or deionized water.

Dissolve $0.03 \mathrm{~g}$ chromium potassium sulphate (chrome alum; $\mathrm{CrK}\left(\mathrm{SO}_{4}\right)_{2} \cdot 12 \mathrm{H}_{2} \mathrm{O}$ in $10 \mathrm{~mL}$ water - wait, do not heat.

Combine the two solutions and pour into the staining tank.

It is advisable to set aside glassware especially for this procedure because it becomes coated with chrome gelatin, which is difficult to remove.
What to do:

1. Put the clean slides in the staining rack. 2. Immerse in the subbing solution for 30 to 60 seconds, with occasional gentle agitation.

3. Lift out and drain off excess liquid, then leave the rack of slides in a dust-free place until dry.

The dry subbed slides are stored in boxes until they are needed. It is convenient to pack them in the cardboard boxes they came in.

\section{Alternative Method}

Make up a more concentrated subbing solution ( $1 \%$ gelatin and $0.1 \%$ chrome alum).

Smear this over one surface of each slide, and leave the slides to dry, lying on the bench and protected from falling dust.

Pack in boxes when dry. 


\section{It's Coming... The New Personal SEM 2000}

Don't miss this exciting event: The New Personal SEM 2000 will be revealed on August 2, 1999 at Microscopy and Microanalysis '99 at the RJ Lee Instrument Booths \#915-919.

This new Microanalysis System (SEM/EDX) has many advancements. One of which is the most technologically advanced software in the industry for increased convenience.

If we tell you too much, then you wouldn't be able to share in our excitement at the show. But more importantly, we want you to personally see the advantages of this new microanalysis system.

Stop by RJ Lee Instruments' Booths 915-919. It's the one booth you don't want to miss at Microscopy and Microanalysis ‘99. 
Strategies for Preventing Detachment of Sections from Glass Slides

Continued from page 22

\section{Recommended Reading}

The following books include detailed discussions of methods for promoting the adhesion of sections to slides.

Culling, C. F A., Allison, R. T. and Barr, W. T. 1985. Cellular Pathology Technique. 4th ed. Butterworths, London.

Gabe, M. 1976. Histological Techniques (English ed., transl. E. Blackith \& A. Kavoor). Masson, Paris.

Kiernan, J. A. 1999. Histological and Histochemical Methods: Theory and Practice. 3rd ed. Butterworth-Heinemann, Oxford.

Lillie, R. D. and Fullmer, H. M. 1976. Histopathologic Technic and Practical Histochemistry. 4th ed. McGraw-Hill, New York.

Table 3: Enclosing Slides in a

Nitrocellulose Film

General Considerations: This method

is applicable to any kind of sections

mounted on slides. Paraffin sections

must be dewaxed and placed in $100 \%$

alcohol (ethanol, methanol or isopropa-

nol). Frozen or cryostat sections must be

dehydrated.

\section{What you need:}

1. Slides with mounted sections, in

$100 \%$ alcohol.

2. Ether-alcohol:

Diethyl ether (anaesthetic ether is suitable): $500 \mathrm{~mL}$

Ethanol (100\%): $500 \mathrm{~mL}$

3. $0.5 \%$ Nitrocellulose

This contains $2.5 \mathrm{~g}$ of nitrocellulose in $500 \mathrm{~mL}$ of ether-alcohol.

It may be made from a solid form of nitrocellulose (such as parlodion) or by dilution of a more concentrated solution. Commercial LVN (low viscosity nitrocelluose) is sold as a $20 \%$ solution.

The $0.5 \%$ solution can be kept for a few years, but see safety note below.

4. $70 \%$ alcohol.

The alcohol concentration is not critical. Add 30 volumes of water to 70 volumes of $100 \%$ or $95 \%$ ethanol.

Safety Note: Nitrocellullose solutions are highly inflammable and great care should be taken

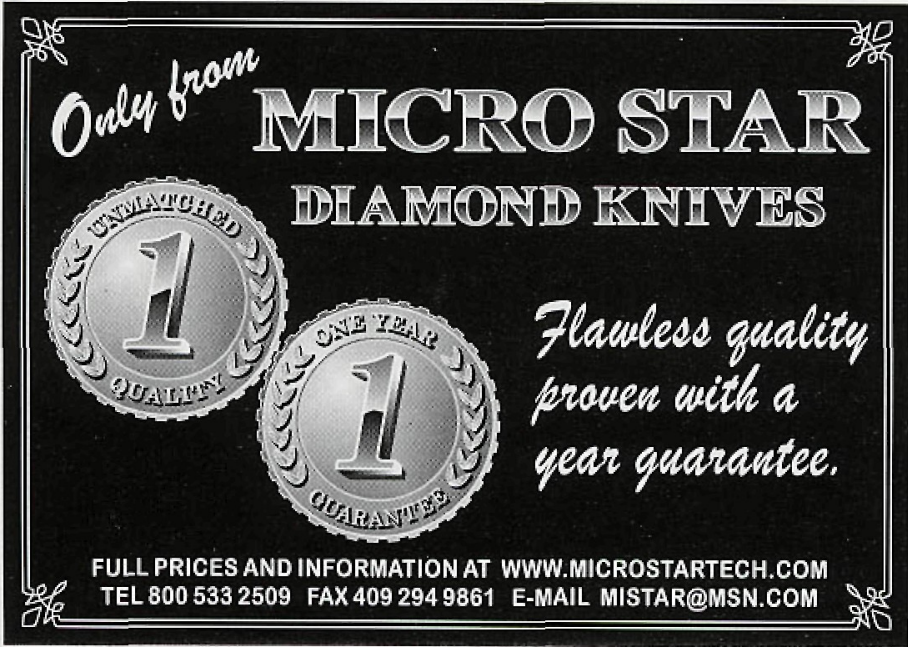

\section{Ruthenium Tetroxide:}

A Complementary Fixative

and Stain to Osmium Tetroxide

Henry Eichelberger, Binghamton Universtiy - SUNY

Ruthenium tetroxide $\left(\mathrm{RuO}_{4}\right)$, which is a stronger oxidizing agent than osmium tetroxide $\left(\mathrm{OsO}_{4}\right)$, reacts well with some of the more polar lipids that fail to show a reaction with $\mathrm{OsO}_{4}{ }_{4}$. It has been demonstrated that the use of $\mathrm{RuO}_{4}$ can overcome the failure of $\mathrm{OSO}_{4}$ to visualize epidermal intercellular lamellae ${ }^{2,3}$. $\mathrm{RuO}_{4}$ reacts strongly with both saturated and unsaturated lipid molecules, as well as with proteins, glycogen, and monosaccharides. $\mathrm{RuO}_{4}$-fixed membranes appear thicker than those fixed with $\mathrm{OsO}_{4} \mathrm{do}^{4}$.

$\mathrm{RuO}_{4}$ penetrates tissue very slowly and sometimes uneven, patchy preservation may occur $r^{5}$. The use of vibratome sections is recommended to optimize penetration of the $\mathrm{RuO}_{4}$ fixative ${ }^{6}$.

There are some artifacts of fixation that limit $\mathrm{RuO}_{4}$ as a postfixative. For example, the distinct pattern of keratin bundles I have observed within epithelial cornified cells of $\mathrm{OsO}_{4-}$ postfixed tissue often have a disrupted, chewed-up appearance with $\mathrm{RuO}_{4}$-postfixed tissue. Thus when $\mathrm{RuO}_{4}$ is used as a postfixative with experimental and pathological tissue, I recommend using a complementary postfixative of $1 \% \mathrm{OsO}_{4}$ or $1 \% \mathrm{OsO}_{4}$ with $1.5 \%$ potassium ferrocyanate to fully confirm any interpretations of abnormality. $\mathrm{RuO}_{4}$ is also a useful stain for polymers and their blends ${ }^{7}$.

A mixture of formaldehyde-glutaraldehyde-ruthenium tetroxide has been used as a fixative ${ }^{8}$. A more typical procedure that I have used routinely with success consists of:

1) Prefix with $3 \%$ glutaraldehyde in $0.1 \mathrm{M}$ cacodylate buffer at $\mathrm{pH} 7$ for a minimum of 1 hour at room temperature.

2) Follow with 3 buffer rinses.

3) Postfix with $0.2 \% \mathrm{RuO}_{4}$ in the same buffer at $\mathrm{pH} 7$ for 1 hour at room temperature.

4) Specimens should be rinsed in 3 changes of distilled water before dehydrating in ethanol or acetone.

Care should be taken in handling $\mathrm{RuO}_{4}$. It is a strong oxidizing agent and reacts violently with filter paper and alcohol. It should be protected from UV light and stored in a refrigerator. A separate waste container should be used for disposal of $\mathrm{RuO}_{4}$, which should not be allowed to come in contact with alcohol, ether, benzene, pyridine or other organic compounds. Always handle in a hood. In case of a spillage, use a sodium bisulfite solution to decompose the $\mathrm{RuO}_{4}$ and flush with plenty of water.

$\mathrm{RuO}_{4}$ comes in a $0.5 \%$ aqueous solution that can be obtained from Electron Microscopy Sciences, Fort Washington, PA (www.emsdiasum.com) or Polysciences Inc., Warrington, PA (www.polysciences.com). A $0.67 \%$ aqueous solution can be prepared from a kit supplied by SPI Supplies, West Chester, PA (www.2 spi.com).

1) Gaylarde, P. and I. Sarkany. 1968. Science, 161:1157.

2) Eichelberger, H.H., et al. 1994. Proc. Microscopy and Microanalysis 270 .

3) Swartzendruber, D.C., et al. 1995. J. Inves. Dermatol. 194:417.

4) Peltarri, A. and H.J. Helminen, et al. 1979. Histochem. J. 11:599.

5) Madison, K.C., et al. 1987. J. Inves. Dermatol. 88: 714.

6) van der Meulen J., et al 1996. J. Microsc. 184:67.

7) Trent, J.S. 1984. Macromolecules 17:2930. 